Analele Universităţii de Vest, Timişoara

Seria Matematică - Informatică

LIII, 2, (2015), 81- 98

\title{
Holomorphy of Osborn loops
}

Isere Abednego Orobosa, Adéníran John Olusola, and Jaíyéọlá Tèmítọ́pẹ́ Gbọ́láhàn

\begin{abstract}
Let $(L, \cdot)$ be any loop and let $A(L)$ be a group of automorphisms of $(L, \cdot)$ such that $\alpha$ and $\phi$ are elements of $A(L)$. It is shown that, for all $x, y, z \in L$, the $A(L)$-holomorph $(H, \circ)=$ $H(L)$ of $(L, \cdot)$ is an Osborn loop if and only if $x \alpha\left(y z \cdot x \phi^{-1}\right)=$ $x \alpha\left(y x^{\lambda} \cdot x\right) \cdot z x \phi^{-1}$. Furthermore, it is shown that for all $x \in L$, $H(L)$ is an Osborn loop if and only if $(L, \cdot)$ is an Osborn loop, $(x \alpha$. $\left.x^{\rho}\right) x=x \alpha, x\left(x^{\lambda} \cdot x \phi^{-1}\right)=x \phi^{-1}$ and every pair of automorphisms in $A(L)$ is nuclear (i.e. $\left.x \alpha \cdot x^{\rho}, x^{\lambda} \cdot x \phi \in N(L, \cdot)\right)$. It is shown that if $H(L)$ is an Osborn loop, then $A(L, \cdot)=\mathcal{P}(L, \cdot) \cap \Lambda(L, \cdot) \cap \Phi(L, \cdot) \cap$ $\Psi(L, \cdot)$ and for any $\alpha \in A(L), \alpha=L_{e \pi}=R_{e \varrho}^{-1}$ for some $\pi \in \Phi(L, \cdot)$ and some $\varrho \in \Psi(L, \cdot)$. Some commutative diagrams are deduced by considering isomorphisms among the various groups of regular bijections (whose intersection is $A(L)$ ) and the nucleus of $(L, \cdot)$.
\end{abstract}

AMS Subject Classification (2000). 20N05; secondary 08A05 Keywords. Osborn loops, holomorphy

\section{Introduction}

The holomorph of a loop is a loop according to Bruck [10]. Since then, the concept of holomorphy of loops has caught the attention of some researchers. Interestingly, Adéníran [1] and Robinson [53], Chein and Robinson[12], Adéníran et. al. [3], Chiboka and Solarin [14], [15], Bruck [10], Bruck and Paige [11], Robinson [52], Huthnance [23], Adéníran et. al. [4] and, 
Jaiyéolá and Popoola [34] have respectively studied the holomorphic structures of Bol/Bruck loops, Moufang loops, central loops, conjugacy closed loops, inverse property loops, A-loops, extra loops, weak inverse property loops and generalized Bol loops.

After the discovery of Osborn loops by Osborn [50] and Huthnance [23], Osborn loops were formally introduced and studied by Basarab [5-9], in the $20^{\text {th }}$ century. In this $21^{\text {st }}$ century, the study of Osborn loops was recently revived by Kinyon [42], where he proposed some problems and heart burning questions. Some of this problems and questions have been solved, answered fully or partially or somewhat addressed in Jaiyéọlá et. al. [39-41], Jaiyéolá and Adéníran [35-37] and Jaiyéolá [28,30,31,33]. Some results on the application of Osborn loops to cryptography can be found in Jaiyéolá and Adéníran [38] and Jaiyéolá [29,32].

Some popular varieties of Osborn loops are: extra loops, Moufang loops, CC-loops, universal WIPLs and V.D. loops. Some studies on them can be found in Drápal [18-22], Csörgö and Drápal [17], Csörgő [16], Kinyon and Kunen [43,44], Kinyon et. al. [45]. Some newly constructed Osborn loops can be found in Isere et. al. [24,26,27], Adéníran and Isere [2].

\section{Preliminaries}

Let $G$ be a non-empty set. Define a binary operation $(\cdot)$ on $G$. If $x \cdot y \in G$ for all $x, y \in G$, then the pair $(G, \cdot)$ is called a groupoid or Magma.

If each of the equations:

$$
a \cdot x=b \quad \text { and } \quad y \cdot a=b
$$

has unique solution in $G$ for $x$ and $y$ respectively, then $(G, \cdot)$ is called a quasigroup.

If there exists a unique element $e \in G$ called the identity element such that for all $x \in G, x \cdot e=e \cdot x=x,(G, \cdot)$ is called a loop. We write $x y$ instead of $x \cdot y$, and stipulate that - has lower priority than juxtaposition among factors to be multiplied. For instance, $x \cdot y z$ stands for $x(y z)$.

For a groupoid $(G, \cdot)$, the right translation of $x$ i.e. $R_{x}: G \rightarrow G$ is defined by $y R_{x}=y \cdot x$ while the left translation of $x$ i.e. $L_{x}: G \rightarrow G$ is defined by $y L_{x}=x \cdot y$ for all $x, y \in G$.

It can now be seen that a groupoid $(G, \cdot)$ is a quasigroup if its left and right translation mappings are bijections or permutations. Since the left and right translation mappings of a loop are bijective, then the inverse mappings 
$L_{x}^{-1}$ and $R_{x}^{-1}$ exist. Let

$$
x \backslash y=y L_{x}^{-1} \quad \text { and } \quad x / y=x R_{y}^{-1}
$$

and note that

$$
x \backslash y=z \Longleftrightarrow x \cdot z=y \quad \text { and } \quad x / y=z \Longleftrightarrow z \cdot y=x .
$$

Hence, $(G, \backslash)$ and $(G, /)$ are also quasigroups. Using the operations $(\backslash)$ and $(/)$, the definition of a loop can be stated as follows.

Definition 2.1. A loop $(G, \cdot, /, \backslash, e)$ is a set $G$ together with three binary operations (.), (/), $\backslash$ ) and one nullary operation e such that

(i) $x \cdot(x \backslash y)=y,(y / x) \cdot x=y$ for all $x, y \in G$,

(ii) $x \backslash(x \cdot y)=y,(y \cdot x) / x=y$ for all $x, y \in G$ and

(iii) $x \backslash x=y / y$ or $e \cdot x=x$ for all $x, y \in G$.

We also stipulate that $(/)$ and $(\backslash)$ have higher priority than $(\cdot)$ among factors to be multiplied. For instance, $x \cdot y / z$ and $x \cdot y \backslash z$ stand for $x(y / z)$ and $x \cdot(y \backslash z)$ respectively.

In a loop $(G, \cdot)$ with identity element $e$, the left inverse element of $x \in G$ is the element $x J_{\lambda}=x^{\lambda} \in G$ such that

$$
x^{\lambda} \cdot x=e
$$

while the right inverse element of $x \in G$ is the element $x J_{\rho}=x^{\rho} \in G$ such that

$$
x \cdot x^{\rho}=e .
$$

A loop is called an Osborn loop if it obeys any of the three identities

$$
\begin{gathered}
x(y z \cdot x)=\left(x^{\lambda} \backslash y\right) \cdot z x \\
x(y z \cdot x)=x\left(y x^{\lambda} \cdot x\right) \cdot z x \\
x(y z \cdot x)=x\left(y x \cdot x^{\rho}\right) \cdot z x
\end{gathered}
$$

Given any two sets $X$ and $Y$. The statement ' $f: X \rightarrow Y$ is defined as $f(x)=y, x \in X, y \in Y$ ' will be expressed as ' $f: X \rightarrow Y \uparrow f(x)=y$ '.

Let $(G, \cdot)$ be a loop and let $A, B$ and $C$ be three bijective mappings, that map $G$ onto $G$. The identity mapping on $G$ will be denoted by $I$. The triple $\alpha=(A, B, C)$ is called an autotopism of $(G, \cdot)$ if and only if

$$
x A \cdot y B=(x \cdot y) C \forall x, y \in G .
$$


Such triples form a group $A U T(G, \cdot)$ called the autotopism group of $(G, \cdot)$ under the binary operation of componentwise composition. That is, for $\left(A_{1}, B_{1}, C_{1}\right),\left(A_{2}, B_{2}, C_{2}\right) \in \operatorname{AUT}(G, \cdot),\left(A_{1}, B_{1}, C_{1}\right)\left(A_{2}, B_{2}, C_{2}\right)=$ $\left(A_{1} A_{2}, B_{1} B_{2}, C_{1} C_{2}\right)$.

If $A=B=C$, then $A$ is called an automorphism of the loop $(G, \cdot)$. Such bijections form a group $A U M(G, \cdot)$ called the automorphism group of $(G, \cdot)$. Let $G$ and $H$ be groups such that $\varphi: G \rightarrow H$ is an isomorphism. If $\varphi(g)=h$, then this would be expressed as $g \stackrel{\varphi}{\cong} h$.

Definition 2.2. Let $(Q, \cdot)$ be a loop and $A(Q) \leq A U M(Q, \cdot)$ be a group of automorphisms of the loop $(Q, \cdot)$. Let $H=A(Q) \times Q$. Define $\circ$ on $H$ as

$$
(\alpha, x) \circ(\beta, y)=(\alpha \beta, x \beta \cdot y) \text { for all }(\alpha, x),(\beta, y) \in H \text {. }
$$

$(H, \circ)$ is a loop and is called the A-holomorph of $(Q, \cdot)$.

The right nucleus of $(L, \cdot)$ is defined by $N_{\rho}(L, \cdot)=\{x \in L \mid z y \cdot x=$ $z \cdot y x \forall y, z \in L\}$. The left nucleus of $(L, \cdot)$ is defined by $N_{\lambda}(L, \cdot)=\{x \in$ $L \mid x \cdot y z=x y \cdot z \forall y, z \in L\}$. The middle nucleus of $(L, \cdot)$ is defined by $N_{\mu}(L, \cdot)=\{x \in L \mid z x \cdot y=z \cdot x y \forall y, z \in L\}$. The nucleus of $(L, \cdot)$ is defined by $N(L, \cdot)=N_{\rho}(L, \cdot) \cap N_{\lambda}(L, \cdot) \cap N_{\mu}(L, \cdot)$. The centrum of $(L, \cdot)$ is defined by $C(L, \cdot)=\{a \in L: a x=x a \forall x \in L\}$ while its center is defined by $Z(L, \cdot)=N(L, \cdot) \cap C(L, \cdot)$.

Let $(G, \cdot)$ be a quasigroup. Then

1. a bijection $U$ is called autotopic if there exists $(U, V, W) \in A U T(G, \cdot)$; the set of all such mappings forms a group $\Sigma(G, \cdot)$.

2. a bijection $U$ is called $\rho$-regular if there exists $(I, U, U) \in \operatorname{AUT}(G, \cdot)$; the set of all such mappings forms a group $\mathcal{P}(G, \cdot)$.

3. a bijection $U$ is called $\lambda$-regular if there exists $(U, I, U) \in A U T(G, \cdot)$; the set of all such mappings forms a group $\Lambda(G, \cdot) \leq \Sigma(G, \cdot)$.

4. a bijection $U$ is called $\mu$-regular if there exists a bijection $U^{\prime}$ such that $\left(U, U^{\prime-1}, I\right) \in \operatorname{AUT}(G, \cdot) . U^{\prime}$ is called the adjoint of $U$. The set of all $\mu$-regular mappings forms a group $\Phi(G, \cdot) \leq \Sigma(G, \cdot)$. The set of all adjoint mapping forms a group $\Psi(G, \cdot)$. 
Theorem 2.1. (Jaiyéolá $[34])$ Let $(G, \cdot)$ be a loop. Let

$$
\begin{gathered}
\psi: \mathcal{P}(G, \cdot) \rightarrow N_{\rho}(G, \cdot) \uparrow \psi(U)=e U, \delta: \Lambda(G, \cdot) \rightarrow N_{\lambda}(G, \cdot) \uparrow \delta(U)=e U, \\
\varphi: \Phi(G, \cdot) \rightarrow \Psi(G, \cdot) \uparrow \varphi(U)=U^{\prime}, \sigma: \Phi(G, \cdot) \rightarrow N_{\mu}(G, \cdot) \uparrow \\
\sigma(U)=e U \text { and } \beta: \Psi(G, \cdot) \rightarrow N_{\mu}(G, \cdot) \uparrow \beta\left(U^{\prime}\right)=e U^{\prime} \\
\text { Then, } \mathcal{P}(G, \cdot) \stackrel{\psi}{\cong} N_{\rho}(G, \cdot), \Lambda(G, \cdot) \stackrel{\delta}{\cong} N_{\lambda}(G, \cdot), \Phi(G, \cdot) \stackrel{\varphi}{\cong} \Psi(G, \cdot), \\
\Phi(G, \cdot) \stackrel{\sigma}{\cong} N_{\mu}(G, \cdot), \Psi(G, \cdot) \stackrel{\beta}{\cong} N_{\mu}(G, \cdot) .
\end{gathered}
$$

\section{Main Results}

Theorem 3.1. Let $(L, \cdot)$ be a loop and $A(L)$ be a group of automorphisms of $(L, \cdot)$. Then, the $A(L)$-holomorph $(H, \circ)$ of $(L, \cdot)$ is an Osborn loop if and only if

$$
x \alpha\left(y z \cdot x \phi^{-1}\right)=x \alpha\left(y x^{\lambda} \cdot x\right) \cdot z x \phi^{-1} \forall x, y, z \in L \text { and } \alpha, \phi \in A(L)
$$

Proof. Suppose A(L)-holomorph $(H, \circ)$ of $(L, \cdot)$ is an Osborn loop, then we have

$$
\begin{gathered}
(\alpha, x) \circ\{[(\beta, y) \circ(\gamma, z)] \circ(\alpha, x)\}=(\alpha, x) \circ\left\{\left[(\beta, y) \circ(\alpha, x)^{\lambda}\right] \circ(\alpha, x)\right\}\{(\gamma, z) \circ(\alpha, x)\} \\
\Leftrightarrow(\alpha(\beta \gamma \alpha), x \beta \gamma \alpha[(y \gamma \cdot z) \alpha \cdot x])=(\alpha, x) \circ\left\{\left(\beta \alpha^{-1}, y x^{\lambda} \cdot x\right) \circ(\alpha, x)\right\} \circ(\gamma \alpha, z \alpha \cdot x) \\
\Leftrightarrow(\alpha(\beta \gamma \alpha), x \beta \gamma \alpha[(y \gamma \cdot z) \alpha \cdot x])=\left[(\alpha, x) \circ\left(\beta, y x^{\lambda} \cdot x\right)\right] \circ(\gamma \alpha, z \alpha \cdot x) \\
\Leftrightarrow(\alpha(\beta \gamma \alpha), x \beta \gamma \alpha[(y \gamma \cdot z) \alpha \cdot x])=\left(\alpha(\beta \gamma \alpha),\left(x \beta\left(y x^{\lambda} \cdot x\right)\right) \gamma \alpha \cdot(z \alpha \cdot x)\right) \\
\Leftrightarrow x \beta \gamma \alpha[(y \gamma \cdot z) \alpha \cdot x]=\left(x \beta \cdot\left(y x^{\lambda} \cdot x\right)\right) \gamma \alpha \cdot(z \alpha \cdot x) \Leftrightarrow \\
x \beta \gamma \alpha[(y \gamma \alpha \cdot z \alpha) \cdot x]=\left(x \beta \gamma \alpha\left(\left(y x^{\lambda}\right) \gamma \alpha \cdot x \gamma \alpha\right)\right)(z \alpha \cdot x) \forall x, y, z \in L
\end{gathered}
$$

and $\alpha, \beta, \gamma \in A(L)$. Putting $\phi=\gamma \alpha$, we have

$x \beta \phi[(y \phi \cdot z \alpha) \cdot x]=\left(x \beta \phi \cdot\left(\left(y x^{\lambda}\right) \phi \cdot x \phi\right)\right)(z \alpha \cdot x) \forall x, y, z \in L$ and $\alpha, \beta, \phi \in A(L)$.

Therefore,

$$
x \beta\left[\left(y \cdot z \alpha \phi^{-1}\right) x \phi^{-1}\right]=\left(x \beta \cdot\left(y x^{\lambda} \cdot x\right)\right)\left(z \alpha \phi^{-1} \cdot x \phi^{-1}\right) .
$$

Letting $\bar{x}=x \phi^{-1}$ and $x=\bar{x} \phi, \bar{z}=z \alpha \phi^{-1}$, we obtain

$$
\bar{x} \phi \beta(y \bar{z} \cdot \bar{x})=\bar{x} \phi \beta\left(y(\bar{x} \phi)^{\lambda} \cdot \bar{x} \phi\right) \cdot \bar{z} \bar{x}
$$


Again, since $\phi$ is an automorphism, then letting $\bar{x} \phi=x$ and $\bar{x}=x \phi^{-1}$, and replacing $\bar{z}$ with $z$ and $\beta$ with $\alpha$, we obtain

$$
x \alpha\left(y z \cdot x \phi^{-1}\right)=x \alpha\left(y x^{\lambda} \cdot x\right) \cdot z x \phi^{-1} \forall x, y, z \in L \text { and } \alpha, \phi \in A(L) .
$$

The converse is obtained by reversing the process.

Corollary 3.2. Let $(L, \cdot)$ be a loop, and $A(L)$ be the group of all automorphisms of $L$. Then, the holomorph $(H, \circ)$ of $(L, \cdot)$ is an Osborn loop if and only if

$$
\left(R_{x^{\lambda}} R_{x} L_{x \alpha}, R_{x \phi^{-1}}, R_{x \phi^{-1}} L_{x \alpha}\right)
$$

is an autotopism of $L$ for all $x \in L$ and all $\alpha, \phi \in A(L)$.

Proof. This is a consequence of Theorem 3.1.

Lemma 3.3. Let $A(L)$ be an automorphism group of an Osborn loop $(L, \cdot)$. The holomorph $(H, \circ)$ of $(L, \cdot)$ is Osborn if and only if the triples

$$
\left(L_{x}^{-1} L_{x \alpha}, I, L_{x}^{-1} L_{x \alpha}\right) \text { and }\left(I, R_{x}^{-1} R_{x \phi^{-1}}, L_{x}^{-1} R_{x}^{-1} R_{x \phi^{-1}} L_{x}\right)
$$

are autotopisms of $L$ for all $x \in L$ and all $\alpha \in A(L)$.

Proof. Let

$$
A=\left(R_{x^{\lambda}} R_{x} L_{x \alpha}, R_{x \phi^{-1}}, R_{x \phi^{-1}} L_{x \alpha}\right) \text { and } B=\left(R_{x^{\lambda}} R_{x} L_{x}, R_{x}, R_{x} L_{x}\right)
$$

Since $(L, \cdot)$ is an Osborn loop, $B$ is an autotopism of $L$ for all $x \in L$. The holomorph of $(L, \cdot)$ is an Osborn if and only if $\mathrm{A}$ is autotopism of $L$ (by Corollary 3.2). So, the triple

$$
B^{-1}=\left(L_{x}^{-1} R_{x}^{-1} R_{x^{\lambda}}^{-1}, R_{x}^{-1}, L_{x}^{-1} R_{x}^{-1}\right)
$$

is also an autotopism of $L$ for all $x \in L$. Hence, $(H, \circ)$ is an Osborn loop if and only if

$$
\begin{gathered}
B^{-1} A=\left(L_{x}^{-1} L_{x \alpha}, R_{x}^{-1} R_{x \phi^{-1}}, L_{x}^{-1} R_{x}^{-1} R_{x \phi^{-1}} L_{x \alpha}\right) \in \operatorname{AUT}(L, \cdot) . \\
\text { Thus, } y L_{x}^{-1} L_{x \alpha} \cdot z R_{x}^{-1} R_{x \phi^{-1}}=(y z) L_{x}^{-1} R_{x}^{-1} R_{x \phi^{-1}} L_{x \alpha} \\
\Leftrightarrow[(x \alpha) \cdot(x \backslash y)] \cdot\left[(z / x) \cdot x \phi^{-1}\right]=(x \alpha) \cdot\left\{[x \backslash(y z)] / x \cdot x \phi^{-1}\right\} .
\end{gathered}
$$

Put $\phi=I$ into equation (3.5) to get

$$
\begin{gathered}
{[(x \alpha) \cdot(x \backslash y)] \cdot z=[(x \alpha) \cdot(x \backslash(y z)]} \\
\Leftrightarrow y L_{x}^{-1} L_{x \alpha} \cdot z=(y z) L_{x}^{-1} L_{x \alpha} \Leftrightarrow\left(L_{x}^{-1} L_{x \alpha}, I, L_{x}^{-1} L_{x \alpha}\right) \in \operatorname{AUT}(L, \cdot) .
\end{gathered}
$$

Now, putting $\alpha=I$ into equation (3.5), we obtain

$$
\begin{aligned}
& {[x \cdot(x \backslash y)] \cdot\left[(z / x) \cdot x \phi^{-1}\right]=x \cdot\left\{[x \backslash(y z)] / x \cdot x \phi^{-1}\right\} \Leftrightarrow y \cdot z R_{x}^{-1} R_{x \phi^{-1}}=} \\
& \left\{[x \backslash(y z)] / x \cdot x \phi^{-1}\right\} L_{x} \Leftrightarrow\left(I, R_{x}^{-1} R_{x \phi^{-1}}, L_{x}^{-1} R_{x}^{-1} R_{x \phi^{-1}} L_{x}\right) \in \operatorname{AUT}(L, \cdot) .
\end{aligned}
$$

The converse follows from Theorem 3.1 and Corollary 3.2. 
Theorem 3.4. Let $A(L)$ be an automorphism group of a loop $(L, \cdot)$. The holomorph $(H, \circ)$ of $(L, \cdot)$ is an Osborn loop if and only if:

(i) $(L, \cdot)$ is an Osborn loop,

(ii) $x \alpha \cdot x^{\rho}, x^{\lambda} \cdot x \phi \in N(L, \cdot)$,

(iii) $\left(x \alpha \cdot x^{\rho}\right) x=x \alpha$,

(iv) $x\left(x^{\lambda} \cdot x \phi^{-1}\right)=x \phi^{-1}$,

for every $x, y \in L$ and $\alpha, \phi \in A(L)$.

Proof. (i) Suppose $(H, \circ)$ is an Osborn loop. $(K, \circ)$ is a subloop of $(H, \circ)$ given by $K=\{(I, x): x \in L\}$. Therefore, $(L, \cdot) \cong(K, \circ)$. Since $(K, \circ)$ is an Osborn loop, it follows that $(L, \cdot)$ is an Osborn loop.

(ii) Since $(H, \circ)$ is Osborn, then by Lemma 3.3, we have

$$
\begin{aligned}
\left(L_{x}^{-1} L_{x \alpha}, I,\right. & \left.L_{x}^{-1} L_{x \alpha}\right) \in \operatorname{AUT}(L, \cdot) \\
& \Leftrightarrow y L_{x}^{-1} L_{x \alpha} \cdot z I=(y z) L_{x}^{-1} L_{x \alpha} \text { for all } y, z \in(L, \cdot) .
\end{aligned}
$$

Putting $y=e$ in equation (3.7) gives: $e L_{x}^{-1} L_{x \alpha} \cdot z I=(e z) L_{x}^{-1} L_{x \alpha} \Rightarrow$ $\left(x \alpha \cdot x^{\rho}\right) z=(x \alpha)(x \backslash z)$

$$
\Rightarrow L_{x \alpha \cdot x^{\rho}}=L_{x}^{-1} L_{x \alpha} .
$$

Again, since $\left(I, R_{x}^{-1} R_{x \phi^{-1}}, L_{x}^{-1} R_{x}^{-1} R_{x \phi^{-1}} L_{x}\right) \in A U T(L, \cdot)$, we have

$$
y I \cdot z R_{x}^{-1} R_{x \phi^{-1}}=(y z) L_{x}^{-1} R_{x}^{-1} R_{x \phi^{-1}} L_{x},
$$

putting $z=e$, we have

$$
\begin{aligned}
& y \cdot e R_{x}^{-1} R_{x \phi^{-1}}=(y e) L_{x}^{-1} R_{x}^{-1} R_{x \phi^{-1}} L_{x} \Rightarrow y \cdot\left(x^{\lambda} \cdot x \phi^{-1}\right)= \\
& y L_{x}^{-1} R_{x}^{-1} R_{x \phi^{-1}} L_{x} \Rightarrow R_{x^{\lambda} \cdot x \phi^{-1}}=L_{x}^{-1} R_{x}^{-1} R_{x \phi^{-1}} L_{x} .
\end{aligned}
$$

Also, substituting $y=e$ in equation (3.9), we get

$$
\begin{gathered}
R_{x}^{-1} R_{x \phi^{-1}}=L_{x}^{-1} R_{x}^{-1} R_{x \phi^{-1}} L_{x} \\
\text { so, } R_{x^{\lambda} \cdot x \phi^{-1}}=R_{x}^{-1} R_{x \phi^{-1}}=L_{x}^{-1} R_{x}^{-1} R_{x \phi^{-1}} L_{x} .
\end{gathered}
$$

So, $x \alpha \cdot x^{\rho} \in N_{\lambda}(L, \cdot)$ and $x^{\lambda} \cdot x \phi \in N_{\rho}(L, \cdot)$, hence, $x^{\lambda} \cdot x \phi, x \alpha \cdot x^{\rho} \in N(L, \cdot)$ for all $x \in L$ and all $\alpha, \phi \in A(L)$. 
(iii) From equation (3.8),

$$
L_{x} L_{x \alpha \cdot x^{\rho}}=L_{x \alpha} \Leftrightarrow\left(x \alpha \cdot x^{\rho}\right) \cdot x y=x \alpha \cdot y .
$$

Since $\left(x \alpha \cdot x^{\rho}\right) \in N_{\lambda}(L, \cdot)$, then

$$
\left(x \alpha \cdot x^{\rho}\right) x=x \alpha \text { for all } x \in L, \alpha, \phi \in A(L) .
$$

(iv) From equation (3.12),

$$
R_{x} L_{x} R_{x^{\lambda} \cdot x \phi^{-1}}=R_{x \phi^{-1}} L_{x} \Leftrightarrow(x \cdot y x)\left(x^{\lambda} \cdot x \phi^{-1}\right)=x\left(y \cdot x \phi^{-1}\right) .
$$

Since $x^{\lambda} \cdot x \phi^{-1} \in N_{\rho}(L, \cdot), x \cdot(y x)\left(x^{\lambda} \cdot x \phi^{-1}\right)=x\left(y \cdot x \phi^{-1}\right) \Rightarrow$

$$
x\left(x^{\lambda} \cdot x \phi^{-1}\right)=x \phi^{-1} .
$$

The converse: suppose $(L, \cdot)$ is an Osborn loop such that (ii), (iii) and (iv) hold. We need to show that $(H, \circ)$ is an Osborn loop.

Already, $\left(x \alpha \cdot x^{\rho}\right) x=x \alpha$, thence $\left(x \alpha \cdot x^{\rho}\right) x \cdot y=x \alpha \cdot y$.

Since $x \alpha \cdot x^{\rho} \in N_{\lambda}(L, \cdot),\left(x \alpha \cdot x^{\rho}\right) \cdot x y=\left(x \alpha \cdot x^{\rho}\right) x \cdot y=x \alpha \cdot y \Rightarrow L_{x \alpha \cdot x^{\rho}}=$ $L_{x}^{-1} L_{x \alpha}$. Next, since $\left(x^{\lambda} \cdot x \phi^{-1}\right) \in N_{\rho}(L, \cdot)$, then

$$
\begin{gathered}
x\left(x^{\lambda} \cdot x \phi^{-1}\right)=x \phi^{-1} \Rightarrow(y x)\left(x^{\lambda} \cdot x \phi^{-1}\right)=\left(y \cdot x \phi^{-1}\right) \\
\Rightarrow(x \cdot y x)\left(x^{\lambda} \cdot x \phi^{-1}\right)=x\left(y \cdot x \phi^{-1}\right) \Rightarrow R_{x^{\lambda}} \cdot x \phi^{-1}=L_{x}^{-1} R_{x}^{-1} R_{x \phi^{-1}} L_{x} .
\end{gathered}
$$

Already, $x\left(x^{\lambda} \cdot x \phi^{-1}\right)=x \phi^{-1}$. Since, $x^{\lambda} \cdot x \phi^{-1} \in N(L, \cdot)$, then $y \cdot x\left(x^{\lambda} \cdot x \phi^{-1}\right)=$ $y \cdot x \phi^{-1} \Rightarrow y x \cdot\left(x^{\lambda} \cdot x \phi^{-1}\right)=y \cdot x \phi^{-1} \Rightarrow$

$$
R_{x^{\lambda} \cdot x \phi^{-1}}=R_{x}^{-1} R_{x \phi^{-1}}
$$

Since $x \alpha \cdot x^{\rho} \in N_{\lambda}(L, \cdot)$, then $\left(L_{x}^{-1} L_{x \alpha}, I, L_{x}^{-1} L_{x \alpha}\right) \in A U T(L, \cdot)$.

And also, since $x^{\lambda} \cdot x \phi^{-1} \in N_{\rho}(L, \cdot)$, then $\left(I, R_{x}^{-1} R_{x \phi^{-1}}, L_{x}^{-1} R_{x}^{-1} R_{x \phi^{-1}} L_{x}\right) \in$ $\operatorname{AUT}(L, \cdot)$. Hence, by Lemma 3.3, the holomorph $(H, \circ)$ of $(L, \cdot)$ is an Osborn loop.

Lemma 3.5. Let $A(L)$ be an automorphism group of a loop $(L, \cdot)$. If the holomorph $(H, \circ)$ of $(L, \cdot)$ is an Osborn loop, then the following identities hold:

(1) $\left(x \alpha \cdot x^{\rho}\right) \cdot x y=x \alpha \cdot y ; x \cdot(x \alpha)^{\rho}=\left(x \alpha \cdot x^{\rho}\right)^{\rho}, \quad\left(x \alpha \cdot x^{\rho}\right) x=x \alpha$,

(2) $(x \cdot y x)\left(x^{\lambda} \cdot x \phi^{-1}\right)=x\left(y \cdot x \phi^{-1}\right) ;\left(x \phi^{-1}\right)^{\lambda} \cdot x=\left(x^{\lambda} \cdot x \phi^{-1}\right)^{\lambda}$,

(3) $y x \cdot\left(x^{\lambda} \cdot x \phi^{-1}\right)=y \cdot x \phi^{-1} ; x\left(x^{\lambda} \cdot x \phi^{-1}\right)=x \phi^{-1}$, 
(4) $x\left(y / x^{\lambda} \cdot x \phi^{-1}\right)=(x y) / x \cdot x \phi^{-1} ; x\left(x^{\rho} / x^{\lambda} \cdot x \phi^{-1}\right)=x^{\lambda} \cdot x \phi^{-1}$,

for all $x, y \in L$ and $\alpha, \phi \in A(L)$.

Proof. From Theorem 3.4, we have

(1)

$$
L_{x \alpha \cdot x^{\rho}}=L_{x}^{-1} L_{x \alpha} \Rightarrow\left(x \alpha \cdot x^{\rho}\right) \cdot x y=x \alpha \cdot y
$$

Put $y=(x \alpha)^{\rho}$ in (3.16) to get $\left(x \alpha \cdot x^{\rho}\right)^{\rho}=x \cdot\left(x^{\alpha}\right)^{\rho}$. Putting $y=e$ in (3.16), then $\left(x \alpha \cdot x^{\rho}\right) x=x \alpha$.

(2)

$$
R_{x^{\lambda} \cdot x \phi^{-1}}=L_{x}^{-1} R_{x}^{-1} R_{x \phi^{-1}} L_{x} \Rightarrow(x \cdot y x)\left(x^{\lambda} \cdot x \phi^{-1}\right)=x\left(y \cdot x \phi^{-1}\right)
$$

Put $y=\left(x \phi^{-1}\right)^{\lambda}$ in (3.17) to get $\left(x^{\lambda} \cdot x \phi^{-1}\right)^{\lambda}=\left(x \phi^{-1}\right)^{\lambda} x$.

(3)

$$
R_{x^{\lambda} \cdot x \phi^{-1}}=R_{x}^{-1} R_{x \phi^{-1}} \Rightarrow y x \cdot\left(x^{\lambda} \cdot x \phi^{-1}\right)=y \cdot x \phi^{-1}
$$

Put $y=e$ in (3.17) to get $x\left(x^{\lambda} \cdot x \phi^{-1}\right)=x \phi^{-1}$.

(4) $R_{x}^{-1} R_{x \phi^{-1}}=L_{x}^{-1} R_{x}^{-1} R_{x \phi^{-1}} L_{x} \Rightarrow L_{x} R_{x}^{-1} R_{x \phi^{-1}}=R_{x}^{-1} R_{x \phi^{-1}} L_{x} \Rightarrow x\left(y / x^{\lambda}\right.$. $\left.x \phi^{-1}\right)=(x y) / x \cdot x \phi^{-1}$. Put $y=x^{\rho}$, then $x\left(x^{\rho} / x^{\lambda} \cdot x \phi^{-1}\right)=x^{\lambda} \cdot x \phi^{-1}$.

The proof is complete.

Lemma 3.6. Let $(L, \cdot)$ be a loop. If the A-holomorph $H(L)$ of $L$ is an Osborn loop, then for all $x \in L$ and $\alpha, \phi \in A(L)$.

(a) $\left(L_{x}^{-1} L_{x \alpha}, I, L_{x}^{-1} L_{x \alpha}\right) \in A U T(L, \cdot)$.

(b) $\left(L_{x \alpha \cdot x^{\rho}}, I, L_{x \alpha \cdot x^{\rho}}\right) \in \operatorname{AUT}(L, \cdot)$.

(c) $\left(I, R_{x}^{-1} R_{x \phi^{-1}}, R_{x}^{-1} R_{x \phi^{-1}}\right) \in \operatorname{AUT}(L, \cdot)$.

(d) $\left(I, L_{x}^{-1} R_{x}^{-1} R_{x \phi^{-1}} L_{x}, L_{x}^{-1} R_{x}^{-1} R_{x \phi^{-1}} L_{x}\right) \in \operatorname{AUT}(L, \cdot)$.

(e) $\left(I, R_{x^{\lambda} \cdot x \phi^{-1}}, R_{x^{\lambda} \cdot x \phi^{-1}}\right) \in A U T(L, \cdot)$.

(f) $\left(R_{x \alpha \cdot x^{\rho}}, L_{x \alpha \cdot x^{\rho}}^{-1}, I\right),\left(R_{x^{\lambda} \cdot x \phi^{-1}}, L_{x^{\lambda} \cdot x \phi^{-1}}^{-1}, I\right) \in \operatorname{AUT}(L, \cdot)$.

Proof. (a) Following the steps in the proof of Lemma 3.3, we obtain (a).

(b) Use Theorem 3.4 and the fact that $L_{x \alpha \cdot x^{\rho}}=L_{x}^{-1} L_{x \alpha}$.

(c) Follow the steps in Lemma 3.3 and Theorem 3.4. 
(d) Follow the steps in Lemma 3.3 and Theorem 3.4.

(e) Follow the steps in Lemma 3.3 and Theorem 3.4.

(f) Since $x \alpha \cdot x^{\rho} \in N(L, \cdot)$, obviously, it is in $N_{\mu}(L, \cdot)$. Then:

$$
x\left(x \alpha \cdot x^{\rho}\right) \cdot y=x \cdot\left(x \alpha \cdot x^{\rho}\right) y \Rightarrow x R_{x \alpha \cdot x^{\rho}} \cdot y L_{x \alpha \cdot x^{\rho}}^{-1}=x y
$$

which implies that

$$
\left(R_{x \alpha \cdot x^{\rho}}, L_{x \alpha \cdot x^{\rho}}^{-1}, I\right) \in A U T(L, \cdot) .
$$

Since $x^{\lambda} \cdot x \phi^{-1} \in N(L, \cdot) \Rightarrow x^{\lambda} \cdot x \phi^{-1} \in N_{\mu}(L, \cdot)$, then by definition,

$$
\begin{gathered}
x\left(x^{\lambda} \cdot x \phi^{-1}\right) \cdot y=x \cdot\left(x^{\lambda} \cdot x \phi^{-1}\right) y \Rightarrow x R_{x^{\lambda} \cdot x \phi^{-1}} \cdot y=x \cdot y L_{x^{\lambda} \cdot x \phi^{-1}} \Rightarrow \\
\left(R_{x^{\lambda} \cdot x \phi^{-1}}, L_{x^{\lambda} \cdot x \phi^{-1}}, I\right) \in \operatorname{AUT}(L, \cdot) .
\end{gathered}
$$

That completes the proof.

Corollary 3.7. Let $(L, \cdot)$ be a loop. If the A-holomorph $H(L)$ of $L$ is an Osborn loop, then for all $x \in L$ and $\alpha, \phi \in A(L)$.

(a) $L_{x}^{-1} L_{x \alpha}, L_{x \alpha \cdot x^{\rho}} \in \Lambda(L, \cdot) ; L_{x \alpha} \in L_{x} \Lambda(L, \cdot)$.

(b) $R_{x}^{-1} R_{x \phi^{-1}}, L_{x}^{-1} R_{x}^{-1} R_{x \phi^{-1}} L_{x}, R_{x^{\lambda} \cdot x \phi^{-1}} \in \mathcal{P}(L, \cdot)$;

$R_{x \phi^{-1}} \in R_{x} \mathcal{P}(L, \cdot), R_{x \phi^{-1}} L_{x} \in R_{x} L_{x} \mathcal{P}(L, \cdot)$.

(c) $R_{x \alpha \cdot x^{\rho}}, R_{x^{\lambda} \cdot x \phi^{-1}} \in \Phi(L, \cdot), L_{x \alpha \cdot x^{\rho}}, L_{x^{\lambda} \cdot x \phi^{-1}} \in \Psi(L, \cdot)$.

Proof. Use Lemma 3.6.

Theorem 3.8. Let $L$ be a loop and $H(L)$ its A-holomorph. If $H(L)$ is an Osborn loop, then $A(L, \cdot)=\mathcal{P}(L, \cdot) \cap \Lambda(L, \cdot) \cap \Phi(L, \cdot) \cap \Psi(L, \cdot)$ and for any $\alpha \in A(L), \alpha=L_{e \pi}=R_{e \varrho}^{-1}$ for some $\pi \in \Phi(L, \cdot)$ and some $\varrho \in \Psi(L, \cdot)$

Proof. From Corollary 3.7, $L_{x \alpha} \in L_{x} \Lambda(L, \cdot) \Rightarrow L_{x \alpha}=L_{x} \lambda$ for some $\lambda \in$ $\Lambda(L, \cdot)$. So

$$
x \alpha \cdot y=(x y) \lambda
$$

implies that, $(\alpha, I, \lambda) \in \operatorname{AUT}(L, \cdot) \Rightarrow \alpha=\lambda \Rightarrow \alpha \in \Lambda(L, \cdot)$. Also, $L_{x \alpha \cdot x^{\rho}}=\lambda$.

$R_{x \phi^{-1}} \in R_{x} \mathcal{P}(L, \cdot)$ implies that $R_{x \phi^{-1}}=R_{x \rho} \Rightarrow y \cdot x \phi^{-1}=(y x) \rho \Rightarrow$ $\left(I, \phi^{-1}, \rho\right) \in \operatorname{AUT}(L, \cdot) \Rightarrow \phi^{-1}=\rho \Rightarrow \phi \in \mathcal{P}(L, \cdot)$.

Next, $R_{x \alpha \cdot x^{\rho}} \in \Phi(L, \cdot) \Rightarrow y R_{x \alpha \cdot x^{\rho}}=y \pi$ for some $\pi \in \Phi(L, \cdot) \Rightarrow y(x \alpha$. $\left.x^{\rho}\right) \cdot x y=y \pi \cdot x y \Rightarrow y(x \alpha \cdot y)=y \pi \cdot x y$. Putting $y=e, e \cdot(x \alpha \cdot e)=e \pi \cdot x e \Rightarrow$ $\alpha=L_{e \pi}$. 
Next, $L_{x \alpha \cdot x^{\rho}} \in \Psi(L, \cdot) \Rightarrow y L_{x \alpha \cdot x^{\rho}}=y \varrho$ for some $\varrho \in \Psi(L, \cdot) \Rightarrow\left(x \alpha \cdot x^{\rho}\right)$. $x y=(x y) \varrho \Rightarrow x \alpha \cdot y=(x y) \varrho$.

Thus, $(\alpha, I, \varrho) \in \operatorname{AUT}(L, \cdot) \Rightarrow \alpha=\varrho=\lambda$. Also, $R_{x^{\lambda} \cdot x \phi^{-1}} \in \Phi(L, \cdot) \Rightarrow$ $R_{x^{\lambda} \cdot x \phi^{-1}}=\pi \Rightarrow y\left(x^{\lambda} \cdot x \phi^{-1}\right)=y \pi \Rightarrow(y x)\left(x^{\lambda} \cdot x \phi^{-1}\right)=(y x) \pi\left(y \cdot x \phi^{-1}=\right.$ $(y x) \pi \Rightarrow \Rightarrow\left(I, \phi^{-1}, \pi\right) \in \operatorname{AUT}(L, \cdot) \Rightarrow \phi^{-1}=\pi=\rho$. So, $\phi \in \Phi(L, \cdot)$.

Finally, $L_{x^{\lambda} \cdot x \phi^{-1}} \in \Psi(L, \cdot) \Rightarrow y L_{x^{\lambda} \cdot x \phi^{-1}}=y \varrho \Rightarrow(y x)\left(x^{\lambda} \cdot x \phi^{-1}\right) y=$ $y x \cdot y \varrho \Rightarrow\left(y \cdot x \phi^{-1}\right) y=y x \cdot y \varrho$.

With $y=e$, then $\left(e \cdot x \phi^{-1}\right) e=e x \cdot e \varrho \Rightarrow \phi^{-1}=R_{e \varrho} \Rightarrow \phi=R_{e \varrho}^{-1}$.

Since $\alpha=\varrho=\lambda$ and $\phi^{-1}=\pi=\rho$ and $\alpha$ and $\phi$ are arbitrary elements from $A(L)$, then $\alpha \in \Lambda(L, \cdot), \alpha \in \mathcal{P}(L, \cdot), \alpha \in \Phi(L, \cdot)$ and $\alpha \in \Psi(L, \cdot)$. Hence,

$$
A(L)=\mathcal{P}(L, \cdot) \cap \Lambda(L, \cdot) \cap \Phi(L, \cdot) \cap \Psi(L, \cdot)
$$

For any $\alpha \in A(L, \cdot), \alpha=L_{e \Phi}=R_{e \Psi}^{-1}$ for some $\pi \in \Phi(L, \cdot)$ and some $\varrho \in$ $\Psi(L, \cdot)$.

Theorem 3.9. Let $L$ be a loop with an A-holomorph Osborn loop $H(L)$. Then for all $x \in L$ and $\alpha, \phi \in A(L)$.

(a) $L_{x}^{-1} L_{x \alpha}=L_{x \alpha \cdot x^{\rho}} \stackrel{\delta, \beta}{\cong} x \alpha \cdot x^{\rho}$.

(b) $R_{x}^{-1} R_{x \phi^{-1}}=L_{x}^{-1} R_{x}^{-1} R_{x \phi^{-1}} L_{x}=R_{x^{\lambda} \cdot x \phi^{-1}} \stackrel{\psi, \sigma}{\cong} x^{\lambda} \cdot x \phi^{-1}=x\left(x^{\rho} / x \cdot x \phi^{-1}\right)$.

(c) $R_{x}^{-1} R_{x \phi^{-1}}=R_{x \alpha \cdot x^{\rho}} \stackrel{\sigma}{\cong} x \alpha \cdot x^{\rho}, L_{x^{\lambda} \cdot x \phi^{-1}} \stackrel{\beta}{\cong} x^{\lambda} \cdot x \phi^{-1}$.

(d) $R_{x}^{-1} R_{x \phi^{-1}}=R_{x \alpha \cdot x^{\rho}} \stackrel{\varphi}{\cong} L_{x \alpha \cdot x^{\rho}}, R_{x^{\lambda} \cdot x \phi^{-1}} \stackrel{\varphi}{\cong} L_{x^{\lambda} \cdot x \phi^{-1}}$.

Proof. Let $U=L_{x}^{-1} L_{x \alpha} \in \Lambda(L, \cdot)$, so $\delta(U)=e U=e L_{x}^{-1} L_{x \alpha}=x^{\rho} L_{x \alpha}=$ $x \alpha \cdot x^{\rho} \in N_{\lambda}(L, \cdot)$. Thus, $L_{x}^{-1} L_{x \alpha} \stackrel{\delta}{\cong} x \alpha \cdot x^{\rho} \forall x \in L, \alpha \in A(L)$.

Let $U=L_{x \alpha \cdot x^{\rho}} \in \Lambda(L, \cdot)$, then, $\delta(U)=e U=e L_{x \alpha \cdot x^{\rho}}=x \alpha \cdot x^{\rho} \in N_{\lambda}(L, \cdot)$.

Thus, $L_{x \alpha \cdot x^{\rho}} \stackrel{\delta}{\cong} x \alpha \cdot x^{\rho} \forall x \in L, \alpha \in A(L)$.

Let $U=R_{x}^{-1} R_{x \phi^{-1}} \in \mathcal{P}(L, \cdot)$, then, $\psi(U)=e U=e R_{x}^{-1} R_{x \phi^{-1}}=x^{\lambda} R_{x \phi^{-1}}=$ $x^{\lambda} \cdot x \phi^{-1} \in N_{\rho}(L, \cdot)$. Thus, $R_{x}^{-1} R_{x \phi^{-1}} \stackrel{\psi}{\cong} x^{\lambda} \cdot x \phi^{-1} \forall x \in L, \phi \in A(L)$.

Let $U=L_{x}^{-1} R_{x}^{-1} R_{x \phi^{-1}} L_{x} \in \mathcal{P}(L, \cdot)$, so $\psi(U)=e U=e L_{x}^{-1} R_{x}^{-1} R_{x \phi^{-1}} L_{x}=$ $x\left(x^{\rho} / x \cdot x \phi^{-1}\right) \in N_{\rho}(L, \cdot)$. Thus, $L_{x}^{-1} R_{x}^{-1} R_{x \phi^{-1}} L_{x} \stackrel{\psi}{\cong} x\left(x^{\rho} / x \cdot x \phi^{-1}\right)$.

Let $U=R_{x^{\lambda} \cdot x \phi^{-1}} \in \mathcal{P}(L, \cdot)$. So, $\psi(U)=e U=e R_{x^{\lambda} \cdot x \phi^{-1}}=x^{\lambda} \cdot x \phi^{-1} \in N_{\rho}(L)$. Thus $R_{x^{\lambda} \cdot x \phi^{-1}} \stackrel{\mathcal{\psi}}{\cong} x^{\lambda} \cdot x \phi^{-1}$.

Let $U=R_{x \alpha \cdot x^{\rho}} \in \Phi(L, \cdot)$, so $\sigma(U)=e R_{x \alpha \cdot x^{\rho}}=x \alpha \cdot x^{\rho} \in N_{\mu}(L, \cdot)$. Thus, $R_{x \alpha \cdot x^{\rho}} \stackrel{\sigma}{\cong} x \alpha \cdot x^{\rho}$. 
Let $U=R_{x^{\lambda} \cdot x \phi^{-1}} \in \Phi(L, \cdot)$, so, $\sigma(U)=e R_{x^{\lambda} \cdot x \phi^{-1}}^{-1}=x^{\lambda} \cdot x \phi \in N_{\mu}(L, \cdot)$. Thus, $R_{x^{\lambda} \cdot x \phi^{-1}} \stackrel{\sigma}{\cong} x^{\lambda} \cdot x \phi^{-1}$.

Let $U=L_{x \alpha \cdot x^{\rho}} \in \Psi(L, \cdot)$, so, $\beta(U)=e L_{x \alpha \cdot x^{\rho}}=x \alpha \cdot x^{\rho} \in N_{\mu}(L, \cdot)$. Thus, $L_{x \alpha \cdot x^{\rho}} \stackrel{\beta}{\cong} x \alpha \cdot x^{\rho}$.

Let $U=L_{x^{\lambda} \cdot x \phi^{-1}} \in \Psi(L, \cdot)$, so, $\beta(U)=e L_{x^{\lambda} \cdot x \phi^{-1}}=x^{\lambda} \cdot x \phi^{-1} \in N_{\mu}(L, \cdot)$. Thus, $L_{x^{\lambda} \cdot x \phi^{-1}} \stackrel{\beta}{\cong} x^{\lambda} \cdot x \phi^{-1}$.

Let $U=R_{x \alpha \cdot x^{\rho}} \in \Phi(L, \cdot)$. So, $\varphi(U)=U^{\prime}=L_{x \alpha \cdot x^{\rho}}$. Thus, $R_{x \alpha \cdot x^{\rho}} \stackrel{\varphi}{\cong} L_{x \alpha \cdot x^{\rho}} \in$ $\Psi(L, \cdot)$.

Let $U=R_{x^{\lambda} \cdot x \phi^{-1}} \in \Phi(L, \cdot)$. So, $\varphi(U)=U^{\prime}=L_{x^{\lambda} \cdot x \phi^{-1}}$. Thus, $R_{x^{\lambda} \cdot x \phi^{-1}} \stackrel{\stackrel{\varphi}{\cong}}{ }$ $L_{x^{\lambda} \cdot x \phi^{-1}} \in \Psi(L, \cdot)$.

Theorem 3.10. Let $(L, \cdot)$ be loop with an A-holomorph Osborn loop $H(L)$. Then,

(a)

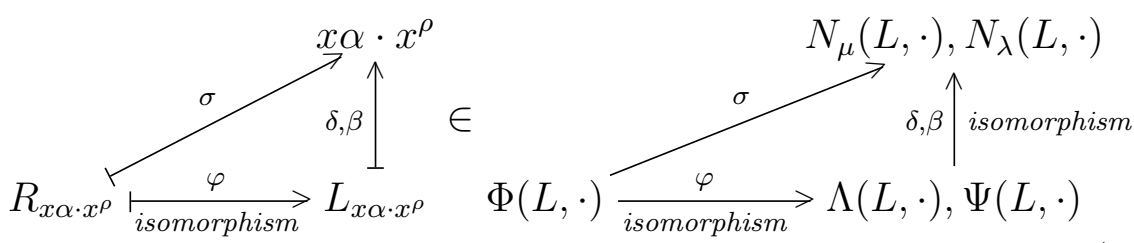

for all $x \in L, \alpha \in A(L)$, i.e. $\sigma=\varphi \delta$ and $\sigma=\varphi \beta$.

(b)

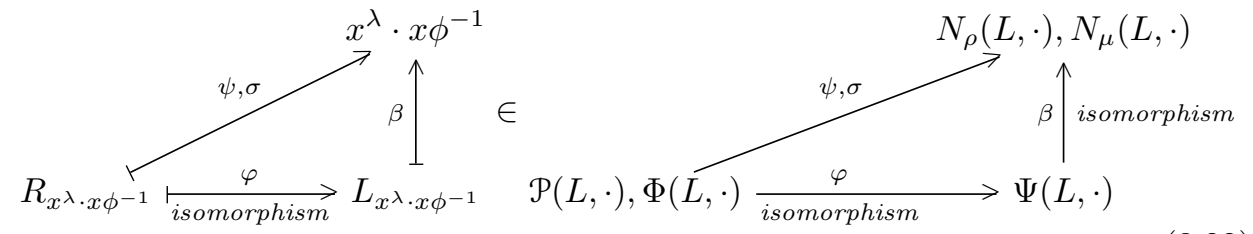

for all $x \in L, \phi \in A(L)$, i.e. $\sigma=\varphi \beta$ and $\psi=\varphi \beta$.

Proof. The proof follows from Theorem 3.9.

Theorem 3.11. Let $(L, \cdot)$ be a loop with an A-holomorph Osborn loop $H(L)$.

(a) The commutative diagram

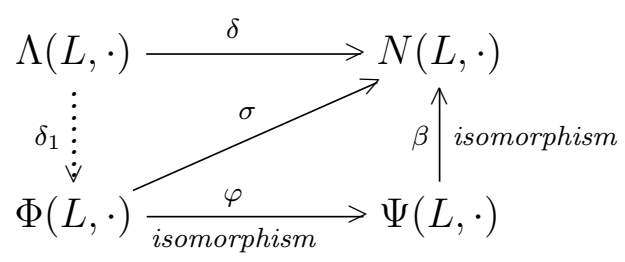


is true and so, $\delta=\delta_{1} \sigma=\delta_{1} \varphi \beta, L_{x \alpha \cdot x^{\rho}} \stackrel{\delta_{1}}{\cong} R_{x \alpha \cdot x^{\rho}}$ and $x \alpha \cdot x^{\rho} \in Z(L, \cdot)$ for all $x \in L, \alpha \in A(L)$.

(b) The commutative diagram

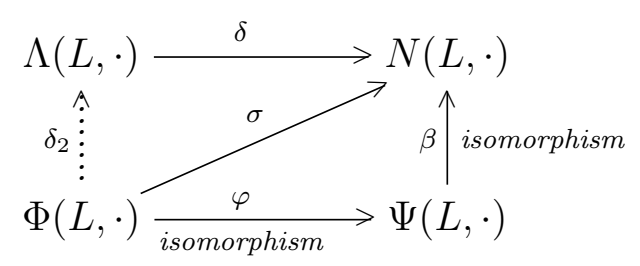

is true and so, $\sigma=\delta_{2} \delta$ and $\delta_{2} \delta=\varphi \beta$ and $R_{x \alpha \cdot x^{\rho}} \stackrel{\delta_{1}}{\cong} L_{x \alpha \cdot x^{\rho}}$.

(c) The commutative diagram

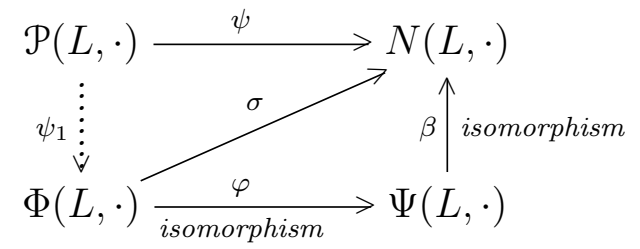

is true and so, $\psi=\psi_{1} \sigma=\psi_{1} \varphi \beta$ and $R_{x^{\lambda} \cdot x \phi^{-1}} \stackrel{\psi_{1}}{\cong} R_{x^{\lambda} \cdot x \phi^{-1}}$.

(d) The commutative diagram

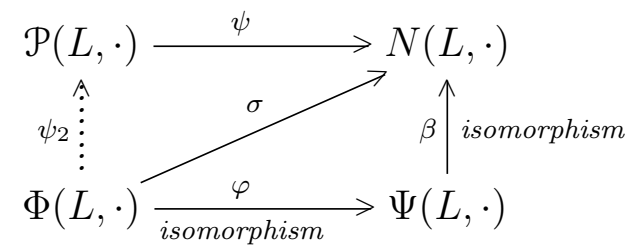

is true and so, $\sigma=\psi_{2} \psi$ and $\psi_{2} \psi=\varphi \beta$ and $R_{x^{\lambda} \cdot x \phi^{-1}} \stackrel{\psi_{2}}{\cong} R_{x^{\lambda} \cdot x \phi^{-1}}$.

(e) The commutative diagram

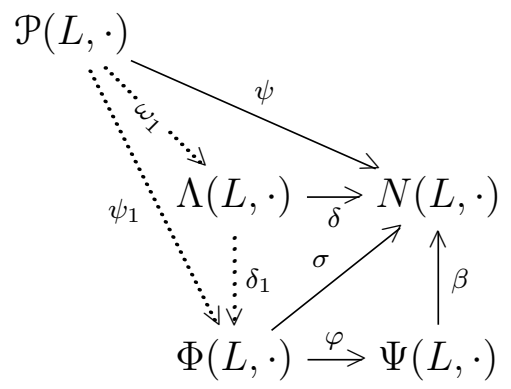

is true and so, $\psi_{1}=\omega_{1} \delta_{1}$ and $\psi=\omega_{1} \delta$ and $R_{x^{\lambda} \cdot x \phi^{-1}} \stackrel{\omega_{1}}{\cong} L_{x^{\lambda} \cdot x \phi^{-1}}$. 
(f) The commutative diagram

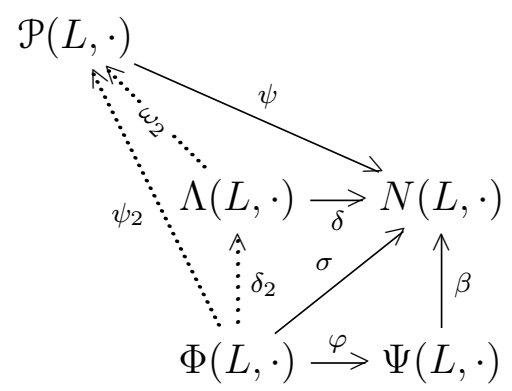

is true and so, $\psi_{2}=\delta_{2} \omega_{2}$ and $\delta=\omega_{2} \psi$, and $L_{x^{\lambda} \cdot x \phi^{-1}} \stackrel{\omega_{2}}{\cong} R_{x^{\lambda} \cdot x \phi^{-1}}$.

Proof. The proof is a consequence of Theorem 3.9.

Corollary 3.12. Let $L$ be a loop with an A-holomorph Osborn loop $H(L)$.

(a) The commutative diagram

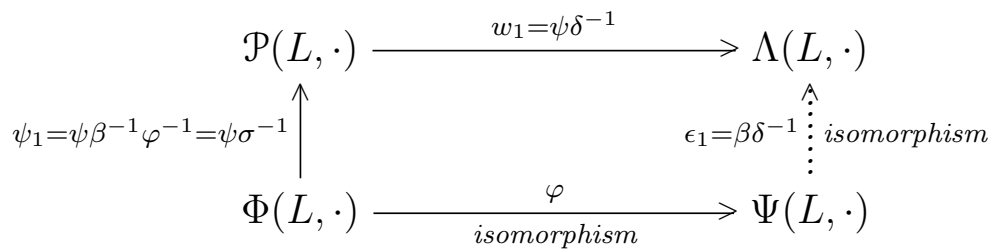

is true and $L_{x^{\lambda} \cdot x \phi^{-1}} \stackrel{\epsilon_{1}}{\cong} L_{x^{\lambda} \cdot x \phi^{-1}}$.

(b) The commutative diagram

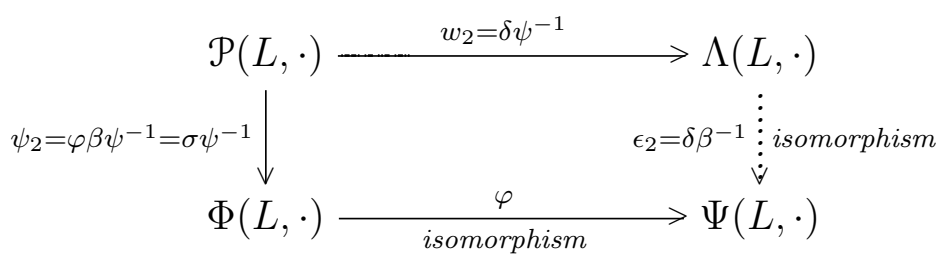

is true and $L_{x^{\lambda} \cdot x \phi^{-1}} \stackrel{\epsilon_{2}}{\cong} L_{x^{\lambda} \cdot x \phi^{-1}}$.

Proof. The proof follows from Theorem 3.9 and Theorem 3.10. $\psi=\psi_{1} \varphi \beta \Rightarrow$ $\psi_{1}=\psi \beta^{-1} \varphi^{-1} \cdot \beta=\epsilon_{1} \delta \Rightarrow \epsilon_{1}=\beta \delta^{-1} . w_{1}=\psi_{1} \varphi \epsilon=\psi \delta^{-1} . w_{2} \psi=\delta \Rightarrow w_{2}=$ $\delta \psi^{-1} . \psi_{2} \varphi=\varphi \beta \Rightarrow \psi_{2}=\varphi \beta \psi^{-1}$. 


\section{Acknowledgement}

The first author wishes to express his profound gratitude and appreciation to the Management of Education Trust Found Academic Staff Training and Development-2009(ETF AST and D)for the grant given him to carry out this Research, as well as, to the management of Ambrose Alli University, Nigeria for their joint support of the grant.

\section{References}

[1] J. O. Adéníran, On holomorphic theory of a class of left Bol loops, Al.I.Cuza, 51(1), (2005), 23-28

[2] J. O. Adéníran and A. O. Isere, Nuclear Automorphism of a class of Osborn Loops, Journal of the Nigerian Association of Mathematical Physics, 22, (2012), 5-8

[3] J. O. Adéníran, Y. T. Oyebo, and D. Mohammed, On certain isotopic maps of central loops, Proyecciones Journal of Mathematics, 30(3), (2012), 303-318

[4] J. O. Adéníran, T. G. Jaiyéolá, and K. A. Idowu, Holomorph of generalized Bol loops, Novi Sad Journal of Mathematics, 44 (1), (2014), 37-51

[5] A. S. Basarab, The Osborn loop, Studies in the theory of quasigroups and loops, Shtiintsa, Kishinev, 193, (1973), 12-18

[6] A. S. Basarab, Osborn's G-loop, Quasigroups and Related Systems, 1(1), (1994), $51-56$

[7] A. S. Basarab, Generalised Moufang G-loops, Quasigroups and Related Systems, 3, (1996), 1-6

[8] A. S. Basarab and A.I. Belioglo, UAI Osborn loops, Quasigroups and loops, Mat. Issled., 51, (1979), 8-16

[9] A. S. Basarab and A. I. Belioglo, Univeral automorphic inverse Gloops, Quasigroups and loops, Mat. Issled., 71, (1979), 3-7

[10] R. H. Bruck, Contributions to the theory of loops, Trans. Amer. Math. Soc., 55, (1944), 245-354

[11] R. H. Bruck and L. J. Paige, Loops whose inner mappings are automorphisms, The annuals of Mathematics, 63(2), (1956), 308-323

[12] O. Chein and D. A. Robinson, An extra law for characterizing Moufang loops, Proc. Amer. Math. Soc., 33, (1972), 29-32

[13] V. O. Chiboka, The study of properties and construction of certain finite order G-loops, Ph.D thesis, Obafemi Awolowo University, Ile-Ife, 1990

[14] V. O. Chiboka and A. R. T. Solarin, Holomorphs of conjugacy closed loops, Scientific Annals of Al.I.Cuza. Univ., 37(3), (1991), 277-284 
[15] V. O. Chiboka and A. R. T. Solarin, Autotopism characterization of G-loops, Scientific Annals of Al.I.Cuza. Univ., 39(1), (1993), 19-26

[16] P. Csörgö, Extending the structural homomorphism of LCC loops, Comment. Math. Univ. Carolinae, 46(3), (2005), 385-389

[17] P. Csörgö and A. Drápal, Left conjugacy closed loops of nilpotency class 2, Results Math., 47, (2005), 242-265

[18] A. Drápal, Conjugacy closed loops and their multiplication groups, J. Alg., 272, (2004), 838-850

[19] A. Drápal, On multiplicatipon groups of left conjugacy closed loops, Comment. Math. Univ. Carolinae, 45, (2004), 223-236

[20] A. Drápal, On left conjugacy closed loops with a nucleus of index two, Abh. Math. Sem. Univ. Hamburg, 74, (2004), 205-221

[21] A. Drápal, Structural interactions of conjugacy closed loops, Trans. Amer. Math. Soc., 360, (2008), 671-689

[22] A. Drápal, On extraspecial left conjugacy closed loops, J. Alg., 302(2), (2006), 771-792

[23] E. D. Huthnance Jr., A theory of generalised Moufang loops, Ph.D. thesis, Georgia Institute of Technology, (1968)

[24] A. O. Isere, S. A. Akinleye, and J. O. Adéníran, On Osborn loops of Order 4n, Acta Universitatis Apulensis Maths. Inform. No., 37, (2014), 31-44

[25] A. O. Isere, J. O. Adéníran, and T. G. Jaiyéolá, Generalized Osborn Loops of Order 4n, Acta Universitatis Apulensis Maths. Inform. No., 43, (2015), 19-31

[26] A. O. Isere, J. O. Adéníran, and T. G. Jaiyéọlá, Classification of Osborn loops of order 4n, Algebras, Groups and Geometries, to appear

[27] A. O. Isere, J. O. Adéníran, and A. R. T. Solarin, Some examples of finite Osborn loops, Journal of Nigerian Mathematical Society, 31, (2012), 91-106

[28] T. G. Jaiyéolá, The study of the universality of Osborn loops, Ph.D. thesis, University of Agriculture, 2008

Abeokuta.

[29] T. G. Jaiyéọlá, On Three Cryptographic Identities in Left Universal Osborn Loops, Journal of Discrete Mathematical Science and Cryptography, 14(1), (2011), 33-50

(DOI:10.1080/09720529.2011.10698322)

[30] T. G. Jaiyéolá, Osborn loops and their universality, Scientific Annals of "Al.I. Cuza" University of Iasi., Tomul, 58(2), (2012), 437-452

[31] T. G. Jaiyéọlá, New identities in universal Osborn loops II, Algebras, Groups and Geometries, 30(1), (2013), 111-126

[32] T. G. Jaiyéolá, On Two Cryptographic Identities in Universal Osborn Loops, Journal of Discrete Mathematical Sciences and Cryptography, 16(2-3), (2013), 95-116

(DOI:10.1080/09720529.2013.821371) 
[33] T. G. Jaiyéolá, On some simplicial complexes of universal Osborn loops, Analele Universitatii De Vest Din Timisoara, Seria Matematica-Informatica, 52(1), (2014), 65-79

(DOI: 10.2478/awutm-2014-0005)

[34] T. G. Jaiyéolá and B. A. Popoola, Holomorph of generalized Bol loops II, Discussiones Mathematicae-General Algebra and Applications, 35(1), (2015), 59-78 (doi:10.7151/dmgaa.1234)

[35] T. G. Jaiyéọlá and J. O. Adéníran, New identities in universal Osborn loops, Quasigroups and Related Systems, Moldova, 179(1), (2009), 55-76

[36] T. G. Jaiyéolá and J. O. Adéníran, Not Every Osborn loop is universal, Acta Math. Acad. Paed. Nvireguhaziensis, 25, (2009), 189-190

[37] T. G. Jaiyéolá and J. O. Adéníran, Loops that are isomorphic to their Osborn loop isotopes(G-Osborn loops), Octogon Mathematical Magazine, 19(2), (2011), 328348

[38] T. G. Jaiyéolá and J. O. Adéníran, On Another Two Cryptographic Identities In Universal Osborn Loops, Surveys in Mathematics and its Applications, 5, (2010), 17-34

[39] T. G. Jaiyéolá, J. O. Adéníran, and A. R. T. Sòlárìn, The universality of Osborn loops, Acta Universitatis Apulensis Mathematics-Informatics, 26, (2011), 301320

[40] T. G. Jaiyéọlá, J. O. Adéníran, and A. R. T. Sòlárìn, Some necessary conditions for the existence of a finite Osborn loop with trivial nucleus, Algebras, Groups and Geometries, 28(4), (2011), 363-380

[41] T. G. Jaiyéọlá, J. O. Adéníran, and A. A. A. Agboọlá, On the second Bryant Schneider group of universal Osborn loops, Societatea Română de Matematică Aplicată si Industrială Journal (ROMAI J.), 9(1), (2013), 37-50

[42] M. K. Kinyon, A survey of Osborn loops, Milehigh conference on loops, quasigroups and non-associative systems, University of Denver, Denver, Colorado, (2005)

[43] M. K. Kinyon and K. Kunen, The structure of extra loops, Quasigroups and Related Systems, 12, (2004), 39-60

[44] M. K. Kinyon and K. Kunen, Power-associative conjugacy closed loops, J. Alg., 304(2), (2006), 679-711

[45] M. K. Kinyon, K. Kunen, and J. D. Phillips, Diassociativity in conjugacy closed loops, Comm. Alg., 32, (2004), 767-786

[46] M.K. Kinyon, K. Kunen, and J.D. Phillips, A generalisation of Moufang and Steiner loops, Alg. Univer., 48(1), (2002), 81-101

[47] K. Kunen, G-loops and Permutation Groups, J. Alg., 220, (1999), 694-708

[48] K. Kunen, The structure of conjugacy closed loops, Trans. Amer. Math. Soc., 352, (2000), 2889-2911

[49] J. M. Osborn, A theorem on A-loops, Proc. Amer. Math. Soc., 9, (1959), 347-349 
[50] J. M. Osborn, Loops with the weak inverse property, Pac. J. Math., 10, (1961), 295-304

[51] H. O. Pflugfelder, Quasigroups and loops : Introduction, Sigma series in Pure Math. 7, Heldermann Verlag, Berlin, 1990

[52] D. A. Robinson, Bol loops, Ph. D thesis, University of Wisconsin, 1964

[53] D. A. Robinson, Holomorphic theory of extra loops, Publ. Math. Debrecen, 18, (1971), 59-64

Isere Abednego Orobosa

Department of Mathematics, Ambrose Alli University,

Ekpoma, 310001, Nigeria

E-mail: abednis@yahoo.co.uk,isereao@aauekpoma.edu.ng

Adéníran John Olusola

Department of Mathematics, Federal University of Agriculture,

Abeokuta 110101, Nigeria

and

UNESCO Professorial Chair of Mathematics (Algebra), National Mathematical Centre,

Abuja, Nigeria.

E-mail: ekenedilichineke@yahoo.com,adeniranoj@unaab.edu.ng

Jaíyéọlá Tèmítọ́pẹ́ Gbọ́láhàn

Department of Mathematics, Obafemi Awolowo University,

Ile Ife 220005 ,

Nigeria

E-mail: jaiyeolatemitope@yahoo.com,tjayeola@oauife.edu.ng

Department of Mathematics and Statistics, Bowen University,

Iwo, Nigeria

E-mail: jaiyeola.temitope@bowenuniversity-edu.org

Received: 2.01.2015

Accepted: 14.01 .2016 